\title{
Prediction of time to ignition in multiple vehicle fire spread experiments
}

\begin{abstract}
This paper describes the application of the flux-time product ignition criterion and the point source flame radiation model to predict the time to ignition in multiple vehicle spread scenarios. Ten experiments from the literature have been selected due to sufficiency of information required to apply the methods. The outcome of this work is to be applied to a risk-based model for the design of car parking buildings to determine when and if a fire spreads between vehicles; therefore, the analysis suggests properties of a representative material that can reasonably account for those external vehicle components that are most likely to ignite first. The application of both methods to the complex problem of multiple vehicle ignition requires several assumptions and simplifications which are discussed in the paper.
\end{abstract}

Keyword: Car parking; Flux time product; Ignition; Point source; Vehicle 